\title{
LA ESTRUCTURA DE OPORTUNIDADES EMPRESARIALES EN LOS DESTINOS TURÍSTICOS DE SOL Y PLAYA. EL CASO DE FUERTEVENTURA (CANARIAS)
}

\author{
J. Rosa Marrero Rodríguez \\ Agustín Santana Talavera \\ Universidad de La Laguna
}

\section{RESUMEN}

El propósito de este trabajo es analizar la desigual estructura de oportunidades en el desarrollo de la actividad empresarial. En concreto, se profundiza en una situación donde los inmigrantes europeos encuentran mejores oportunidades que los residentes nacionales, al contrario de lo que se describe habitualmente en la literatura sobre el emprendimiento de los inmigrantes y la economía étnica. Para ello, se tomará como objeto material de estudio los destinos turísticos de sol y playa, en particular la isla canaria de Fuerteventura, y a partir de datos secundarios y entrevistas en profundidad. Resulta relevante en este trabajo la combinación coherente de diversas aproximaciones teóricas para el análisis de estos procesos de desarrollo empresarial.

Palabras clave: turismo; migraciones; lifestyle migration; iniciativa empresarial; Fuerteventura.

\section{Structure of opportunities for entrepreneurs in the touristic destinations. Fuerteventura island in the Canary Islands}

\section{ABSTRACT}

The purpose of this paper is to analyze the unequal structure of opportunities in the development of economic activity. It is a specific case where European immigrants have better opportunities than the local residents. This is an unusual situation because the

Fecha de recepción:

Fecha de aceptación:

Departamento de Sociología y Antropología. Universidad de La Laguna. Campus de Guajara. 38200 San Cristóbal de la Laguna. SANTA CRUZ DE TENERIFE (España).E-mail: jrmarrod@ull.es, asantana@ull.edu.es 
tradicional literature on the entrepreneurship of immigrants and ethnic economy. The study was carried out in Fuerteventura, a sun and beach touristic destination in the Canary Islands. The research used secondary data and interviews in depth. In this work, it's important the coherent combination of different theoretical approaches for the analysis of these business development processes.

Keywords: tourism; migrations; lifestyle migration; entrepreneurship; Fuerteventura.

\section{INTRODUCCIÓN}

El propósito de este trabajo es analizar la desigual estructura de oportunidades en el desarrollo de la actividad empresarial. En concreto, interesa profundizar en una situación donde los inmigrantes de origen europeo encuentran mejores oportunidades empresariales que los residentes nacionales, al contrario de lo que se describe habitualmente en la literatura sobre el emprendimiento de los inmigrantes y la economía étnica. Para ello, se tomará como objeto material de estudio los destinos turísticos de sol y playa ${ }^{1}$.

Desde los años noventa del siglo XX, los destinos turísticos masivos de sol y playa se vienen enfrentando a cambios en las características de la demanda, dando paso a una potencial reorganización en los componentes de la oferta. Consumidores más exigentes, mejor informados y polifacéticos, conviven con agencias online que cuestionan la intermediación tradicional, y compañías aéreas low cost que facilitan el viaje a nuevos grupos (Ávila Bercial y Barrado Timón, 2005; Cooper y Hall, 2008; González y Ruiz, 2006; Gil y Romero, 2008). Más allá de alojamiento y playas, los destinos se ven en la tesitura de complementar su oferta, y renovar el producto. Algunos autores lo han denominado estrategia de singularización (Vera Rebollo y Baños Castiñeira, 2010), en la que sería fundamental mantener la demanda, captar nuevos segmentos de clientes, e incrementar el gasto turístico en destino; situándose en una fase de renovación de destinos turísticos plenamente posfordista (Agarwal, 2002; Ioannides y Debbage, 1997; Smeral, 1998). Sin embargo, dichos cambios distan de ser mecánicos, pues estas empresas requieren estructuras, know-how y cultura relativamente distintas de las ya establecidas.

La isla canaria de Fuerteventura es el espacio donde se ha realizado esta investigación (Morales et al., 2012). Tras más de dos décadas de crecimiento, se ha consolidado como destino turístico de sol y playa, recibiendo más de dos millones de turistas al año. Dicho crecimiento ha sido espectacular, cercano al $200 \%$ desde comienzos de los años noventa (mientras que en el conjunto del Archipiélago canario era del 70\%). En 2009 la isla fue declarada Reserva de la Biosfera por la UNESCO, en un territorio que ya contaba con diversos mecanismos de protección. Esta nueva figura cubre todo el territorio insular, estando concebida para contribuir a un mejor engarce entre crecimiento económico y protección medioambiental.

1 Este trabajo se ha llevado en el marco del proyecto "Diseño de escenarios óptimos de gobernanza turística en Reservas de la Biosfera", dirigido por Agustín Santana Talavera, y financiado por el Ministerio de Ciencia e Innovación. 
En este trabajo interesa el análisis de la iniciativa empresarial centrada en actividades deportivo-recreativas marítimas, en particular las empresas que ofertan buceo, surf, windsurf y kitesurf (Latiesa y Paniza, 2006; Martín Rojo y Paláez Verdet, 2006; Simôes Brasileiro, 2006); que se han convertido en los últimos años en un icono del turismo insular. Se trata de analizar cómo se desenvuelven las relaciones entre agentes públicos y privados para la consolidación de nuevas actividades que vienen a complejizar el producto sol y playa, a través de un enriquecimiento de los productos vinculados al mar y al deporte. En particular, interesan las características y competencias que presentan estos nuevos empresarios, y en las relaciones que entablan con el resto del entramado productivo turístico local. El aspecto central a tratar se refiere a la importancia que los inmigrantes de origen europeo tienen en la renovación del destino, puesto que se ha creado una estructura de oportunidades que les favorece. De manera adicional, se podrá conocer también cómo los cambios en los destinos turísticos tienen lugar gracias a la confluencia de procesos de distinta naturaleza.

Las hipótesis centrales son dos. Por un lado, este emprendimiento y cualificación en la oferta de Fuerteventura parte, en gran medida, de una dinámica generada por la misma actividad turística; pero no desde su oferta sino desde su demanda. Las particulares características de esta actividad económica favorecen que sean los propios consumidores, convertidos luego en empresarios, los que contribuyen a cualificar la oferta. Como segunda hipótesis, se plantea que la estructura de oportunidades es más favorable a los residentes procedentes de distintos países europeos que a los locales. Dichas oportunidades surgen de la mayor probabilidad por parte de los extranjeros de contar con credenciales y conocimientos específicos para desarrollar las actividades. Todo ello en un contexto de complejas relaciones público-privadas.

\section{PLANTEAMIENTOS TEÓRICOS, OBJETIVOS Y METODOLOGÍA}

La investigación relativa al emprendimiento en los destinos turísticos por parte de los propios turistas no es nueva (Lardiés, 1999); esto es así porque los desplazamientos vinculados al bienestar personal y a ciertos estilos de vida han favorecido nuevos tipos de negocio en muchos destinos turísticos. Así, para Peters et al. (2009), en el sector turístico el emprendimiento está más vinculado al estilo de vida que a la profesionalización racional, al buscar los individuos unas determinadas condiciones de vida. Este planteamiento es claramente distinto al schumpeteriano, dado que estos empresarios suelen tener una mayor motivación por la calidad de vida y menos por la rentabilidad; lo que favorece la subutilización de recursos y capitales, un management poco racional e innovador, entre otros. Como veremos, no es exactamente el caso de los residentes extranjeros estudiados.

En un marco más general, el concepto de Lifestyle Migration ha sido importante para los trabajos sobre los desplazamientos del norte al sur de Europa, en una mezcla de comportamientos entre el turismo y la residencia, teniendo también importancia la migración laboral en sentido estricto. Por ejemplo, O’Reilly plantea la importancia de la dimensión de la elección personal y su relación con conceptos como la calidad de vida y el estilo de vida (O’Reilly, 2000; Benson y O’Reilly, 2009). 
Giner Monfort (2013) plantea como insuficiente esta formulación teórica para comprender el fenómeno de la migración residencial. A partir de comienzos del siglo XX, en zonas tradicionales de turismo residencial centradas en personas de edad avanzada, se observa un rejuvenecimiento de la población de extranjeros, incorporándose muchos de ellos al mercado laboral local. Para el autor, "en muchos aspectos, la realidad de estos negocios se aproxima al concepto de mercado étnico, tal y como se maneja éste para designar los comercios de la población extranjera" (p. 146). Esta idea conectaría, como queremos desarrollar aquí, la literatura sobre la economía étnica con la orientada al Lifestyle Migration.

Por otro lado, González Enríquez (2008) ha planteado que los extranjeros comunitarios presentan progresivamente una estructura de edad menos envejecida, dado el aumento de activos en la misma; también que los factores económicos no son tan importantes en la decisión de establecerse en España, y que sus niveles educativos son superiores a la media española, al contrario que otros colectivos de inmigrantes.

Más recientemente, Huete et al. (2013) han venido a profundizar en las limitaciones que presenta la aproximación teórica del Lifestyle Migration para el estudio de los inmigrantes europeos. Dichas limitaciones tendrían que ver con una aproximación al objeto de estudio a través de las motivaciones y dimensiones subjetivas del mismo, obviando las dimensiones objetivas. Atendiendo al colectivo de británicos en la Costa Mediterránea española, habría que considerar que coinciden las motivaciones vinculadas al estilo de vida con las económicas propiamente dichas. Y que tanto migrantes en busca de un determinado estilo de vida como los desplazados por motivos económicos, buscan ambos una mejora de su vida. Lo cierto es que las dimensiones objetivas y las subjetivas son importantes para comprender fenómenos como el desplazamiento de europeos u otros grupos, sean recreativos o laborales. A partir de las consecuencias de la crisis económica española de mitad de los 2000, muestran la debilidad del enfoque del Lifestyle Migration, centrado en dimensiones subjetivas y en la selección a priori de nacionalidades concretas, y mucho menos en los comportamientos reales de los individuos, y en las consecuencias que generan en los lugares donde se instalan.

También O’Reilly (2012) ha retomado este asunto. La migración es un proceso, no un evento. Y sólo el análisis combinado de los condicionantes estructurales y de las percepciones, habitus y relaciones entre individuos a lo largo del tiempo puede ayudar a comprender estos procesos sociales de movilidad entre países. Por lo que también es importante considerar que los migrantes están localizados en redes, organizaciones y comunidades (véase también Stone y Stubbs, 2007; Membrado Tena, 2015).

Estas reflexiones nos conducen a otro campo de análisis, por lo habitual independiente en términos académicos, la que se refiere a la actividad empresarial de los inmigrantes. Parecería que hay dos tradiciones, una orientada al análisis de los desplazamientos de personas desde los países desarrollados, y otra dedicada a los movimientos desde los países menos desarrollados. Cuando resulta que estamos en ambos casos, ante movimientos migratorios.

En el caso de las formulaciones desde la economía étnica, hay que tener en cuenta varias aproximaciones teóricas. Por un lado, la propuesta culturalista, que ve en las dimensiones culturales del grupo inmigrante los componentes explicativos de su quehacer emprendedor. Luego está el enfoque de la estructura de oportunidades, que pone más énfa- 
sis en el contexto y los factores situacionales. Más tarde, se habla de enfoque integrador, que tiene en cuenta las aproximaciones anteriores de forma conjunta (Solé et al., 2007).

Una línea de trabajo que parte de esta literatura académica, y de interés en este trabajo, es la dimensión transnacional de la migración: la perspectiva desde la óptica del estado nacional impide en ocasiones detenerse en los vínculos que los inmigrantes mantienen con los países de procedencia, a veces durante generaciones. Para el caso español, muchas de estas aproximaciones se han centrado en colectivos nacionales procedentes de países menos desarrollados (Solé et al., 2007).

Para Arjona Garrido y Checa Olmos (2006a), a las tres aproximaciones teóricas mencionadas, hay que sumar la teoría de la incrustación o encajado mixto desarrollada en Europa, debido al complicado ajuste que las propuestas anteriores tienen en el caso europeo. Y es que en el espacio europeo deben abordarse estos asuntos incluyendo los marcos jurídico-normativos como elementos claves de la formación de emprendedores étnicos. Aquí tienen cabida las estructuras de oportunidad y también el régimen normativo. Retomando el concepto de transnacionalidad, "los mismos procesos causales que afectan a la relación empresarial étnica, como desventajas estructurales asociadas a la figura de inmigrante no influyen tanto en las actividades transnacionales... en una lógica económica de este tipo se pueden encontrar inmigrantes muy preparados que han abandonado sus trabajos asalariados bien remunerados, para comenzar una actividad empresarial con la que utilizar bien sus habilidades, el biculturalismo y las redes transnacionales para incrementar ganancias materiales" (p. 133).

Por su parte, Jonker et al. (2009) plantean que el emprendimiento tiene mucho que ver con la capacidad inventiva (innovación, creatividad, visión, dedicación y optimismo) y con la posesión de determinadas cualificaciones y competencias; pero lo que es importante de reseñar ahora es su afirmación de que los atributos para el emprendimiento difieren de un sector de actividad a otro.

En este sentido, este trabajo se coloca en la necesaria conexión entre diferentes propuestas teórico-empíricas para el análisis de los emprendedores de origen europeo en los destinos turísticos de sol y playa españoles. Mientras los desarrollos teóricos centrados en la inserción laboral y empresarial de los inmigrantes aportan una mirada sobre los condicionantes objetivos y subjetivos de la misma; por otro lado, resulta fructífero detenerse en las particulares características estructurales que presenta el sector turístico. Pues en la combinatoria de ambas dimensiones podrá comprenderse mejor el proceso central del que se ocupa este trabajo. Se propone abordar conjuntamente una aproximación teórica que incluya una reflexión sobre los componentes que favorecen la actividad empresarial en los inmigrantes y una consideración sobre las particularidades del sector turístico.

Así, se podría considerar que estamos ante un caso en el que se produce una especie de adecuación desequilibrada entre lo que los inmigrantes de origen europeo pueden ofrecer y el tipo de nicho empresarial que resulta ser el sector turístico; y que esta adecuación es mucho más imperfecta cuando se trata de residentes de origen español. En este sentido, Rath y Kloosterman (2000) proponen tener en cuenta los componentes económicos (las condiciones del mercado), geográficos, culturales (especificidades de algunos colectivos étnicos, profusamente estudiado en el caso de las minorías étnicas en países desarrollados, tales como cultura del emprendimiento), pero también las dimensiones sociales (capital social) e institucionales. Los emprendedores operan en un entorno social e institucional, 
por lo que hay que atender a las relaciones entre los negocios de los inmigrantes, el capital social y el contexto general económico e institucional (como son las normas y leyes).

De esta manera, hay que contar con las particularidades del sector turístico. Así, Williams y Shaw (2011) han planteado que hay varios elementos distintivos en la actividad turística, como son la simultaneidad de producción y consumo, la importancia que para la innovación tienen los empleados, los turistas, y el conocimiento de los proveedores; casi más que la propia innovación interna. Esto afectará a las oportunidades que se le presentan a residentes locales y extranjeros. O el planteamiento de autores como Riley et al. (2002) sobre las débiles barreras de entrada que tiene el sector turístico, lo que favorece una más rápida incorporación de los potenciales emprendedores.

Por último, se deriva también de los planteamientos anteriores, la necesaria importancia de las relaciones del emprendedor con su entorno (Herrera Echeverri, 2009), a través del concepto de red social. Aquí el acento está puesto en la importancia de las relaciones formales e informales en la actividad de los emprendedores. En este sentido, este trabajo también participa de la preocupación por las complejas relaciones entre actores privados y públicos en el desarrollo económico-social (Becerra Rodríguez, 2008; Brunet Icart y Galeana Figueroa, 2013; Casanueva Rocha et al., 2010; Diez, 2008; Elorie, 2009; Merinero Rodríguez, 2010; Oliveira et al., 2014). Así, Casanueva Rocha et al. (2010) inciden en las relaciones entre capital social, relaciones interorganizativas e innovación, centrándose en la transmisión de conocimiento tácito. Interesa, además, reseñar una idea interesante en esta investigación: el conocimiento valioso para la innovación depende más de relaciones de calidad con actores que posean recursos, que del número de actores con los que se relacionan las empresas. Por su parte, para García Macías (2002), los lazos fuertes no son siempre determinantes para conformar redes de colaboración interempresas, cobrando aquí validez la tesis de los lazos débiles; y Dredge (2006) enfatiza la importancia de las relaciones entre los ámbitos público y privado para el desarrollo de redes de asociación.

A partir de todo lo anterior, se plantea lo siguiente. Teniendo en cuenta las reflexiones de Huete et al. (2013) sobre los límites que presenta el concepto de 'lifestyle migration', de las aportaciones procedentes de la teoría de la estructura de oportunidades para inmigrantes que incluye los componentes sociales e institucionales, y del planteamiento teórico más general de O’Reilly (2012) sobre la teoría de la estructuración y la migración como proceso; pero también desde una perspectiva que contempla a los empresarios condicionados por un espacio de redes, organizaciones y marco normativo, se proponen los siguientes objetivos.

En primer lugar, analizar el marco general de condicionantes externos de los empresarios dedicados a actividades recreativo-deportivas instalados en Fuerteventura, lo que incluye las características del entramado productivo, y también las relaciones entre el ámbito privado y el público. Se describirá la evolución reciente del sector en esta isla canaria, y el espacio que ocupan las empresas dedicadas a actividades recreativo-deportivas; también habrá una descripción relativa a las relaciones con el resto del entramado productivo y con la administración local. A continuación, se estudiarán las características que presentan los residentes procedentes del norte de Europa, en contraste con los residentes locales, que beneficiarán a los primeros en la puesta en marcha de dichas empresas recreativo-deportivas. En particular, se analizarán las competencias y habilidades que les favorecen (Arjona Garrido y Checa Olmos, 2006b). 
Pese a la diversidad de actividades recreativo-deportivas, nos hemos centrado en las realizadas en torno al mar, en particular, cuatro de ellas: buceo, surf, windsurf y kitesurf.

La metodología empleada es una combinación de datos secundarios y entrevistas en profundidad. Con respecto a las fuentes de datos secundarios, se ha trabajado con información de AENA, Estadísticas de Empleo Registrado, la Encuesta de Gasto Turístico del Gobierno de Canarias, el Padrón Municipal de Habitantes, la Encuesta de Ingresos y Condiciones de Vida de 2013 y la Encuesta de Población Activa. La metodología de todas estas fuentes está disponible en la página web del Instituto de Estadística del Gobierno de Canarias (ISTAC) y/o del Instituto Nacional de Estadística (INE).

En cuanto a las entrevistas, y teniendo en cuenta los objetivos de la investigación, se confeccionó un guión semi-estructurado, luego se contactó a personas de los subsectores empresariales mencionados a través de la estrategia de la 'bola de nieve'. A lo largo del mes de mayo de 2015 se entrevistaron a quince empresarios y profesionales vinculados a la administración local. Dichas entrevistas se transcribieron y analizaron. Así, cuando no se menciona fuente alguna, la información procederá de las entrevistas realizadas.

\section{LAS EMPRESAS DEPORTIVO-RECREATIVAS MARÍTIMAS EN LA ESTRUCTURA ECONÓMICO-PRODUCTIVA DE FUERTEVENTURA}

En Fuerteventura, la actividad turística centrada en sol y playa se ha ido consolidando a lo largo de los últimos veinte años, hasta tal punto que en 2015 recibió 2.186.421 pasajeros. Al tiempo, y progresivamente diversos espacios naturales y bienes culturales se han venido poniendo en valor turístico. En medio de este proceso, hay que destacar la declaración de la isla como Reserva de la Biosfera (Santana et al., 2011). La acción pública contribuye a la diversificación del producto turístico, como lo muestran diversas acciones recientes (inauguración de la red insular de senderos, del mirador astronómico de Sicasumbre, y del observatorio de aves; o la organización de festivales de música). Fuerteventura es un destino consolidado de sol y playa, en proceso de complejización a través de un conjunto diverso de actividades y productos de naturaleza y culturales (Díaz Rodríguez et al., 2012; Santana Talavera et al., 2015). En esta isla es central el sector turístico. Sin embargo, después de 2007 sufre una importante crisis económica, como en el resto del Estado, lo que afecta al conjunto de su economía, aunque especialmente al sector de la construcción; y traduciéndose ello en un importante incremento del desempleo.

Pese al descenso en el número de empresas, las empresas relacionadas con el sector turístico han mantenido un crecimiento relativo, en particular algunos subsectores, entre ellas, las orientadas a las actividades artísticas, deportivas, recreativas y de entretenimiento. Precisamente, las empresas dedicadas a las actividades recreativas y deportivas serán el objeto de estudio de esta investigación.

Así, en una isla con una clara especialización económica centrada en el sector turístico, destaca, entre otros, la progresiva creación de empresas de oferta complementaria. Lo cual tiene que ver con la complejización de la actividad turística. Ocurre, además, que esta especialización turística no arrastra al resto de los sectores económicos, dominando la escena los servicios. Sólo el $10 \%$ de la población trabaja en el sector primario o secundario. En el sector servicios destaca la hostelería, abarcando un tercio de los empleos. 


\section{Gráfico 1 \\ EVOLUCIÓN DE LA ENTRADA DE PASAJEROS NO INTERINSULARES EN LAS PRINCIPALES ISLAS TURÍSTICAS CANARIAS 1993-2015}

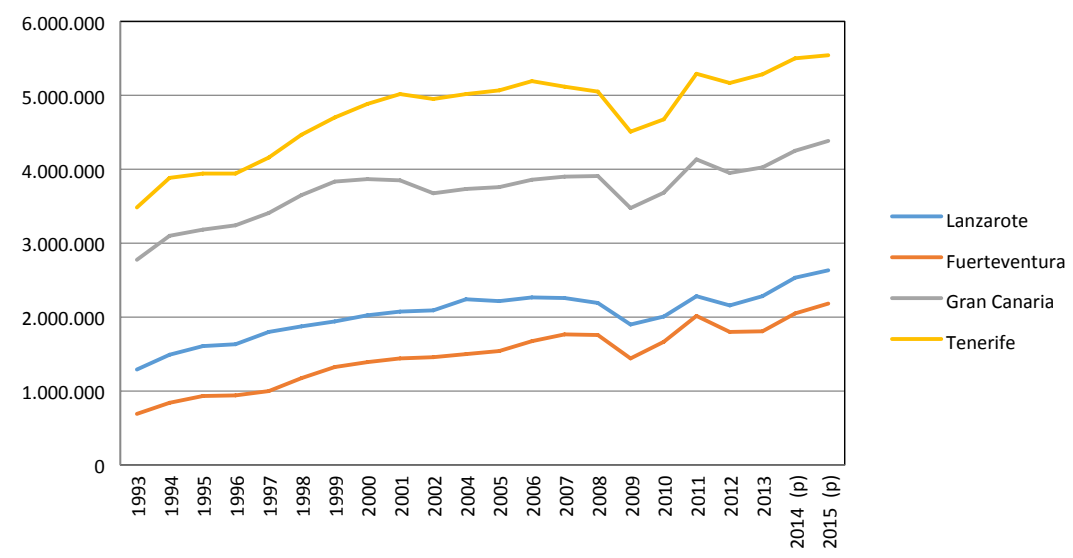

Fuente: AENA.

Las empresas objeto de estudio presentan una serie de características diferenciales con respecto al resto del entramado turístico (alojamiento y restauración). Su número es reducido, en comparación al alojamiento y la restauración (el 2\% del empleo frente al $36 \%$ de hostelería-restauración, gráfico 2); pero en un proceso de crecimiento relativo. Son microempresas, entre 5 y 20 empleados, aunque excepcionalmente alguna llega a los 90 empleados en temporada alta. Por último, es frecuente que estén dirigidas por empresarios procedentes del norte y centro de Europa, que en su mayor parte han accedido a la actividad a partir del amateurismo.

Estas empresas presentan una clara tendencia a la especialización, con una gama limitada de actividades recreativas y deportivas, incluidas las de iniciación. Esto lleva a una diferenciación entre clientes expertos y amateurs. Algunas de estas empresas tienen asociada una tienda de alquiler y venta de materiales para la realización de las actividades (como es el caso del alquiler de trajes de buceo, o de tablas en windsurf); esta parte del negocio de alquiler no es despreciable en el total del volumen de su negocio. Y en ocasiones, ofertan un paquete con alojamiento y transfer, pero tal circunstancia no es frecuente, $\mathrm{y}$ en tal caso se produce cuando se trata de un grupo numeroso.

Se ha dividido a las empresas objeto de estudio en dos grupos, dependiendo de su nivel de autonomía, aunque en cada grupo habrá variaciones según tamaño y grado de informalidad. Las empresas autónomas mantienen un vínculo directo con los clientes, no mediatizado por los Tour Operadores (TO). Esto se debe a un conocimiento directo de la empresa a través de su página web, a la existencia de una clientela repetidora, o porque están vinculadas a algún hotel o cadena a través de la cual acceden a un gran número de clientes potenciales. En algunos casos, los campeonatos deportivos celebrados en la isla les han puesto en el mapa de los demandantes de estas actividades. En esta tesitura, no 
necesitan ni se relacionan con los TO, o lo hacen de manera puntual. En cierta medida, Internet no crea el vínculo, sino que afianza y consolida una relación continuada con los clientes. Las relaciones con hoteles o TO más o menos continuadas en algunos casos no obvia la autonomía que caracteriza a este primer grupo.

\section{Gráfico 2 \\ EMPLEO REGISTRADO SEGÚN RAMAS DE ACTIVIDAD, FUERTEVENTURA SEGUNDO CUATRIMESTRE 2015}

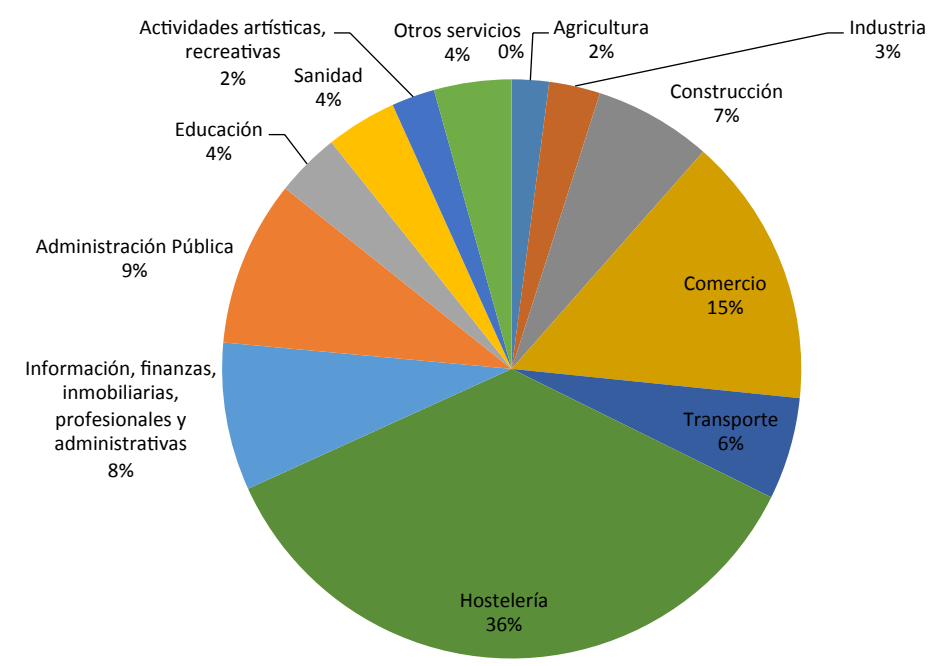

Fuente: Estadística de Empleo Registrado, ISTAC.

El grupo de empresas dependientes se encuentra en otra situación. Es sólo aparente el que el turista esté asequible para ofertarle servicios o productos en destino. Debe superarse el filtro de recepcionistas y otros empleados; y porque más de la mitad de los turistas visita la isla a través de la modalidad del todo incluido. Esto dificulta el acceso al cliente potencial; y, por tanto, facilita una dependencia de los TO, y en menor medida, de los hoteles. Así, la dificultad de acceso a los clientes tiene que ver con la importancia de los TO en la estructura turística insular y en la intermediación, y en un menor conocimiento por parte de los clientes.

Para algunas de las empresas autónomas, ha sido importante la asistencia a ferias, pero sobre todo la celebración de campeonatos de windsurf y kitesurf, que han puesto a la isla en el mapa de la recreación y el deporte. Históricamente, las empresas han sido las pioneras con tales iniciativas, y a posteriori han venido a ser apoyadas por la administración local.

La actividad turística en la isla muestra una pequeña tendencia a la estacionalidad a favor del invierno; pero para estas actividades, la temporada alta transcurre de junio a diciembre. Lo que afecta al número de empleados, que pasa a la mitad en la temporada baja; esto no impide que se observe también un alto grado de repetición de los empleados de una temporada a la siguiente. En cuanto al volumen de clientela anual, se calcula 
que los turistas oscilan entre 6.000 y 30.000, según la actividad y el tamaño de empresa. En apariencia, estamos ante un segmento de turistas que planifican con antelación su viaje; favoreciendo que internet sea un mecanismo importante de reservas y compra.

Las actividades seleccionadas presentan particularidades técnicas. Pues, por la dependencia del mar para su realización, no se pueden desarrollar en cualquier punto de la costa. Por ejemplo, en el kitesurf es aconsejable que la costa sea arenosa y no de piedras, y que el viento vaya de mar a tierra. En cualquier caso, habrá de considerarse la normativa administrativa.

\section{EL ENTRAMADO PRODUCTIVO LOCAL Y LA COMPLEJIDAD NORMATIVA}

Estas empresas se desenvuelven en un entramado productivo local cuyas dimensiones relacionales deben considerarse. Como ya se ha señalado, la centralidad estructural de los TO, pero también las relaciones informales entre empresas y la dimensión transversal de carácter normativo. Con respecto a lo primero, hoteles y complejos de apartamentos alojan a los turistas, lo que constituye el punto de arranque para la captación de los clientes; pero el ámbito del alojamiento no es tan importante como la intermediación, los Tour Operadores. Como se puede observar en los gráficos 3 y 4, casi el 50\% de los vuelos han sido comprados por los turistas a través de TO; si a ello le sumamos las ventas a través de agencias de viaje, casi alcanza las 3/4 partes del total de vuelos.

Esta importancia en la venta de los vuelos, se traslada luego a su centralidad en la dinámica del destino turístico. Efectivamente, algunas de estas empresas pasan a depender de los TO porque muchos de los turistas vienen bajo la fórmula del paquete completo, o del todo incluido; y se les exige una comisión. En caso de recurrir a los hoteles como mecanismo de acceso a los turistas, también. Otra opción que reproduce la centralidad de los TO, es la creación de paquetes con excursiones o actividades incluidas. Desde el punto de vista histórico, aunque algunas de estas empresas crecen a la sombra de los TO, al incrementarse su clientela directa deciden desvincularse de aquellos. Pero para ello se requiere haber adquirido un prestigio.

No obstante, a través de la asociación con un hotel o cadena, algunas empresas han dado con un mecanismo de colaboración más beneficioso. Es frecuente, en estos casos, que las instalaciones de la empresa estén localizadas en dichos hoteles. De igual manera, a veces ofertan un paquete donde se suma la actividad al alojamiento; éste se concierta con hoteles específicos.

En lo relativo a las relaciones entre empresas, se han detectado relaciones cordiales pero débiles, debido al reducido número de acciones conjuntas; casi todos los empresarios se conocen entre sí, y es muy bajo el nivel de asociacionismo formal. Las acciones coordinadas son muy limitadas: bien el reparto informal del territorio insular, como es el caso de las empresas de buceo que acuerdan realizar la actividad sólo en unos pocos lugares, aunque tengan el permiso para bucear en toda la costa insular. En las ocasiones en que los clientes demandan sumergirse en otras zonas de la isla o un determinado tipo de buceo que sólo es posible en otros lugares donde no operan habitualmente, la opción es enviar los clientes a las empresas que ocupan estas otras zonas de la isla. También en ciertas ocasiones se han llevado a cabo promoción conjunta en ferias, y con más regularidad, destaca el intercambio de información, sobre todo el relativo a la normativa y regulación administrativa. 
Por último, las relaciones con la administración local e insular (Cabildos) son frecuentes, aunque con frecuencia conflictivas. En tal sentido, hay que partir de que la política turística, debido a su impacto territorial, se ha centrado casi exclusivamente en el sector alojativo; y de que en unas pocas décadas ha introducido diversos cambios regulatorios y organizativos en la actividad (Almeida García, 2012; Velasco González, 2005). A lo que hay que añadir también la desigual trayectoria regulatoria, que comienza con la indiferencia hacia el sector hasta la actual obsesión reglamentista, bajo la percepción de que su importancia económica así lo demanda; y que, una vez planteada la política regulatoria, ésta no es acompañada sino de precarios mecanismos de inspección y de una reducida relación colaborativa con los actores implicados. Que pretenden ser compensados con nuevas cuotas de regulación.

En la actualidad, uno de los principales problemas para todos los empresarios entrevistados es el marco regulatorio, percibido como complejo, en lo que respecta al desarrollo de actividades vinculadas al mar. Tanto en el caso del buceo como en el surf, windsurf y kitesurf, la actividad se inicia en la costa, tiene lugar en el mar y finaliza de nuevo en tierra. Ello requiere de una normativa de acceso desde la costa (playa, puerto deportivo u otro acceso) cuando se trata de actividades comerciales.

\section{Gráfico 3 \\ TURISTAS SEGÚN CANALES DE VENTA DE LOS VUELOS, FUERTEVENTURA 2014}

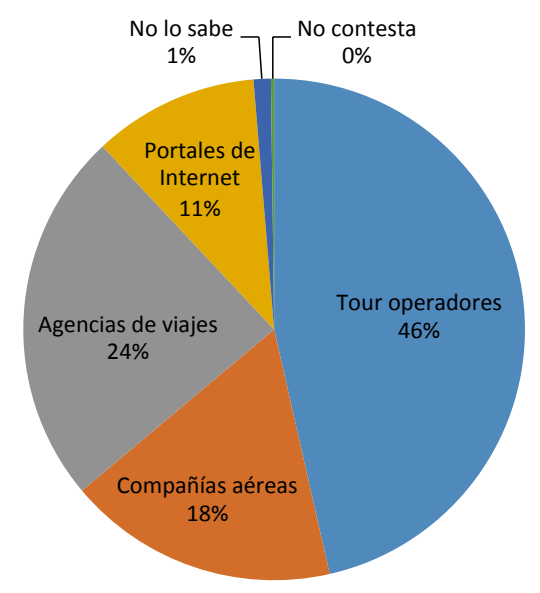

Fuente: Encuesta de Gasto Turístico, ISTAC.

Todas las actividades estudiadas deben obtener diversos permisos para poder realizarse legalmente. Ante la ausencia de una única oficina desde la que tramitar toda la gestión, los empresarios deben acudir a distintas instancias administrativas. En general, es importante el descontento con este tipo de gestiones porque, según las personas consultadas, es complejo, engorroso y poco claro el conjunto de información y documentos a presentar. Por otro lado, es frecuente que los requisitos cambien de un año para otro, o que no coincidan las exigencias de las distintas instancias administrativas implicadas. 
El problema generado es doble. Por un lado, hay un espacio de informalidad surgido tras un largo período donde la ausencia de regulación administrativa era la norma. Y, por otro lado, las exigencias administrativas con frecuencia no están acompañadas de fórmulas de inspección. Ante la continuidad de la informalidad, se incrementan las exigencias, nuevamente no acompañadas de inspección. En este entramado local de relaciones desarrollan su actividad las empresas deportivo-recreativas marítimas.

Así, el pequeño entramado de empresas orientadas a estas actividades marítimas se desenvuelve en un tejido productivo donde los TO tienen una posición central, con relaciones débiles con el resto de empresas y también con la administración local. En este entorno empresarial encuentran una oportunidad más bondadosa los residentes de origen extranjero que los locales.

\section{Gráfico 4 \\ TURISTAS SEGÚN CONCEPTOS PAGADOS EN ORIGEN, FUERTEVENTURA 2014}

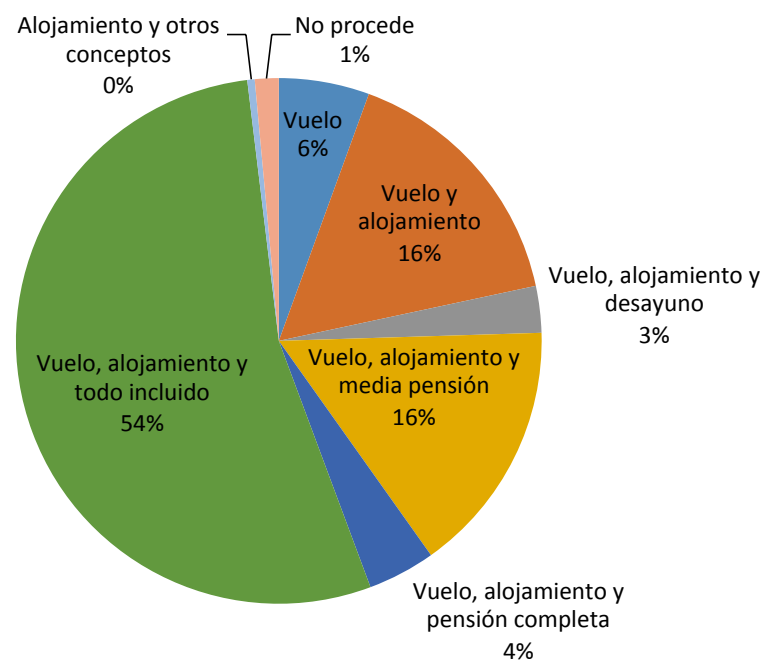

Fuente: Encuesta de Gasto Turístico, ISTAC.

\section{LA ESTRUCTURA DE OPORTUNIDADES PARA LOS EXTRANJEROS DE ORIGEN EUROPEO}

Paralela a la importancia creciente del turismo después de los 80-90, tuvo lugar un importante crecimiento demográfico que ha transformado la estructura social de la isla; desde 1991 hasta 2015, dicho crecimiento fue del 190\% (gráfico 5). Ahora bien, la mayor parte de este crecimiento se ha alimentado de la inmigración, habiéndose convertido en un rasgo distintivo de la estructura demográfica insular. Esto es fundamental para comprender algunas de las características de las empresas estudiadas, dado el origen extranjero de muchos de los empresarios estudiados. Este crecimiento demográfico basado en 
la inmigración hace que la población insular presente una estructura demográfica joven; por otro lado, los niveles educativos medios de la población residente local son bajos, destacando entre otros las reducidas competencias idiomáticas, una barrera para el acceso a las actividades económicas objeto de estudio.

En este contexto debe analizarse la situación de los empresarios entrevistados. En su mayor parte, son de origen extranjero; a lo largo de las últimas dos décadas, y desde el centro y oeste de Europa (alemanes, holandeses, suizos...) se han venido instalando en la isla, y pasado el tiempo ponen en marcha la actividad empresarial. En estas trayectorias personales, el turismo y el deporte han actuado como elementos tractores de estas personas hacia las islas. Luego convierten sus aficiones (y el conocimiento adquirido con las mismas) en capital específico para desarrollar una actividad empresarial; a lo que hay que añadir la cercanía cultural respecto de los turistas, incluido el idioma. Estos tres elementos, unos más que otros, se van a convertir en un hándicap para el emprendimiento de los residentes locales.

\section{Gráfico 5 \\ EVOLUCIÓN DE LA POBLACIÓN EN FUERTEVENTURA, 1991-2015}

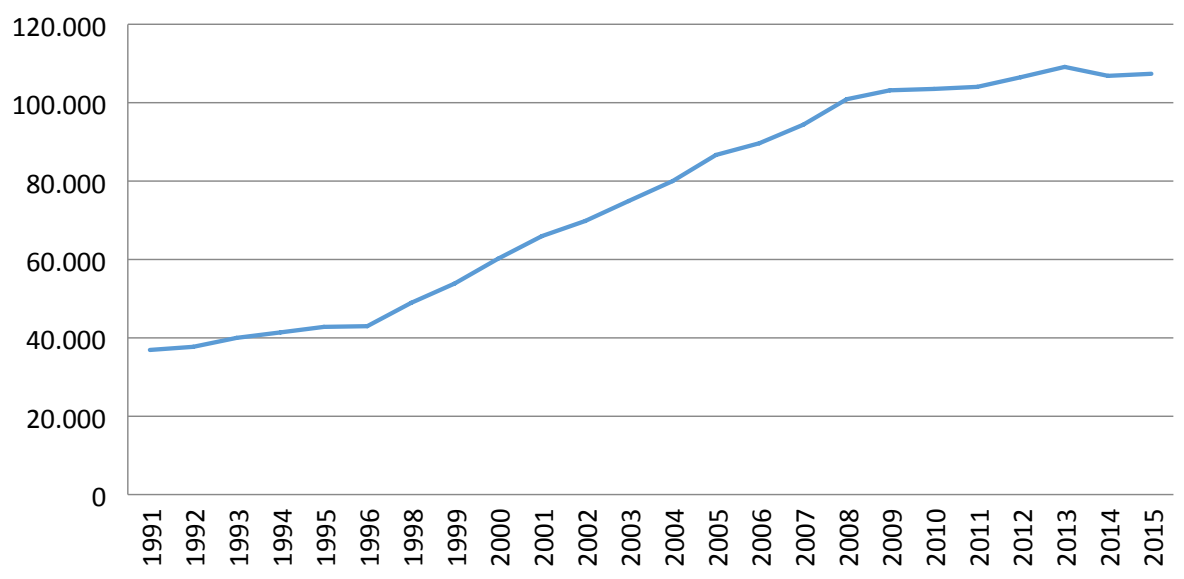

Fuente: Padrón Municipal de Habitantes, ISTAC.

Así, en la constitución de estas trayectorias suman, en primer lugar, la proximidad cultural e idiomática de estos empresarios con los clientes potenciales, pero también, en segundo lugar, las propias características de las actividades implicadas. La práctica continuada en muchos deportes lleva a una especialización que, junto a los certificados pertinentes, son una lanzadera hacia la creación de empresas. Esta práctica no requiere de la adquisición de títulos avalados por el sistema educativo formal, pues se obtienen a través de federaciones reconocidas a nivel internacional. Aquí las titulaciones no funcionan como mecanismo de cierre, pues si fuera así, los residentes locales se verían más favorecidos, como ocurre con otras ocupaciones (Muzio y Kirkpatrick, 2011; Sáez Carreras, 2005; Andrews y Wærness, 2011; Sciulli, 2007). 
En tercer lugar, como bien ha planteado Warde (2005), las propias prácticas se van complejizando, generando nuevas modalidades, que sólo los especialistas pueden captar y poner en marcha. Esta complejización tiene lugar a través de diferentes fórmulas y mecanismos: nuevas actividades, intensificación de esfuerzos o retos, nuevas herramientas o instrumentos (cambiar la marca de la tabla de kitesurf, por ejemplo).

En cuarto lugar, se trata de un espacio dentro del sector turístico que, pese a su complejidad administrativa, presenta unas barreras de entrada más bajas que otras como el sector hotelero. En quinto lugar, en cuanto a los españoles que se incorporan al emprendimiento, se detectan dos características que funciona como hándicap para su exitosa incorporación a las actividades objeto de estudio. Por un lado, la mentalidad generada por el boom constructor-turístico, que favoreció una cultura de rentas rápidas sin grandes inversiones en formación; y que en este nuevo escenario les obliga a negociaciones complejas con los TO, a inversiones inciertas, y a mayor formación. Esto nos lleva al segundo impedimento: la falta de formación en idiomas, para unas actividades en las que resulta crucial la comprensión de todas las instrucciones.

$\mathrm{Y}$ en sexto lugar, una parte de los empresarios y empleados de estas empresas forman parte de una especie de red transnacional desde Europa hasta Canarias. Las competencias en cercanía cultural, en idiomas y en certificaciones relativas a deportes específicos han favorecido dos procesos: por un lado, algunos empleados y empresarios no se han formado en actividades vinculadas al mar sino, por ejemplo, en deportes de invierno; y se reconvierten en Canarias. La familiaridad con deportes en otros entornos turísticos, y la atención a los clientes forman parte de un aprendizaje que luego se aplica en estas actividades. Así, hay instructores que, durante una parte de su trayectoria laboral, hacen un circuito de invierno en Europa con deportes de invierno y en verano con actividades como el windsurf o kitesurf en las Islas.

\section{Gráfico 6 \\ COMPOSICIÓN DE LA POBLACIÓN DE FUERTEVENTURA SEGÚN PAÍSES DE NACIMIENTO, 2015}

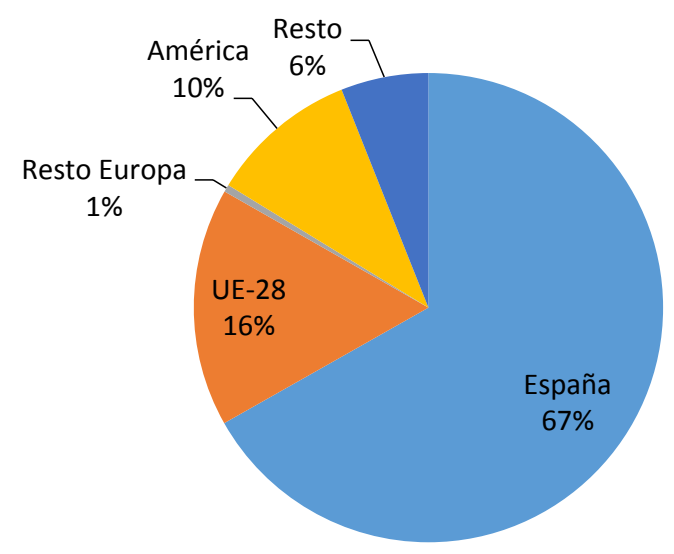

Fuente: Padrón Municipal de Habitantes, ISTAC. 


\section{Gráfico 7 \\ COMPARATIVA DE NIVELES EDUCATIVOS DE LA POBLACIÓN DE 16 Y MÁS AÑOS EN FUERTEVENTURA, CANARIAS Y ESPAÑA}

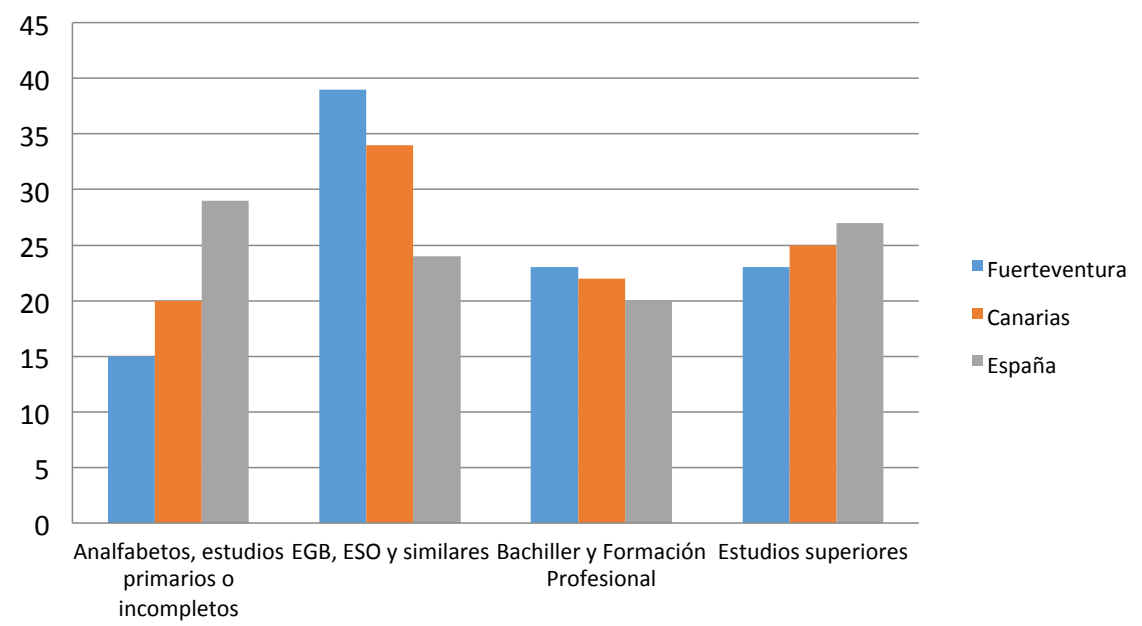

Fuente: Encuesta de Ingresos y Condiciones de Vida 2013 (ISTAC) y Encuesta de Población Activa 2013 (INE).

Y por otro, es frecuente que se incorporen estudiantes extranjeros (alemanes principalmente) con titulaciones relacionadas con los deportes. Los 3-4 meses que pasan en la isla les permite disfrutar de la actividad y aprender; y así, convalidan parte de sus créditos de prácticas. Se da el caso de empresas en las que los instructores con títulos reconocidos forman a practicantes o estudiantes que a posteriori obtendrán el título. Éste último aspecto, se sitúa en un marco institucional derivado de la libre circulación de personas dentro de la Unión Europea. Por tanto, estos extranjeros no presentan la misma discriminación institucional que otros colectivos de inmigrantes no europeos (Solé et al., 2000).

Así pues, se observan dimensiones relativas a las características de los individuos y a la actividad empresarial a desarrollar que en su combinación permiten comprender la mejor posición de los inmigrantes europeos frente a los residentes locales.

\section{CONCLUSIONES}

En este trabajo se ha prestado atención a la estructura de oportunidades que favorece más a unos grupos que a otros en el desarrollo de la actividad empresarial, en particular el beneficio que encuentran los inmigrantes europeos para desarrollar actividades empresariales en determinados entornos turísticos.

En lo que respecta a la iniciativa empresarial centrada en actividades deportivo-recreativas marítimas (buceo, surf, windsurf y kitesurf), las relaciones entre agentes públicos y privados, y entre las distintas empresas orientadas a la actividad turística, no son lo suficientemente colaborativas. Pese a ello, dicha iniciativa se ha desarrollado, estando 
alimentada por dinámicas que poco tienen que ver con la colaboración entre empresas e instituciones. Las propias características de la actividad turística y de su entramado productivo han favorecido que turistas, convertidos en residentes, acaben siendo emprendedores, al rentabilizar el conocimiento adquirido como practicantes de actividades deportivas, el idioma y la cultura que comparten con la clientela turística, y la propia complejización de las actividades deportivo-recreativas. Esta actividad emprendedora no se ve favorecida por las dinámicas internas clásicas de los destinos turísticos de sol y playa, en los que el TO ocupa un papel central en la intermediación y en los procesos internos del destino; pero tampoco por el funcionamiento administrativo local, que legisla mucho e inspecciona y dialoga poco con los actores empresariales.

Estos comportamientos no pueden ser comprendidos desde la perspectiva del Lifestyle Migration. Puesto que se desarrolla una actividad empresarial convencional, y no orientada únicamente a complementar rentas obtenidas en los países de origen. Aquí, el destino turístico crea una estructura de oportunidades que les beneficia e impulsa; al contrario de lo que les ocurre a otros colectivos de inmigrantes no europeos, peor tratados por el mercado laboral español (Martín Artiles et al, 2011; Oso, 2012).

Por otro lado, una parte de este colectivo de residentes de origen europeo parece formar parte de una corriente transnacional de personas en movimiento más o menos regular y periódico entre sus países de origen y Fuerteventura. Bien residiendo una parte del año en España, dedicados a actividades deportivas, bien en formación entre España y Alemania, por ejemplo. Aquí encontramos 'inmigrantes muy preparados... para comenzar una actividad empresarial con la que utilizar bien sus habilidades, el biculturalismo y las redes transnacionales para incrementar ganancias materiales' (Frändberg, 2014; Solé et al., 2007). Y esto probablemente ocurre de una manera que es distinta a otras actividades económicas, con lo que tiene razón Jonker et al. (2009) cuando plantean que 'los atributos para el emprendimiento difieren de un sector de actividad a otro'.

Las relaciones en el interior del entramado productivo local turístico permiten comprender mejor los procesos y relaciones entre empresas, sus limitaciones y ventajas. Con lo que la literatura dedicada a las redes sociales ha aportado una perspectiva muy fructífera a este trabajo. Las redes informales benefician especialmente a las empresas objeto de estudio, mientras que las formales les son menos rentables, excepción hecha de aquellas empresas que colaboran con un hotel o cadena en particular. En cualquier caso, parece que lo que predominan son relaciones débiles: los actores se relacionan poco y puntualmente entre ellos.

Tomando como punto de partida enfoques holísticos como el empleado aquí, las características de los empresarios, y las del contexto productivo e institucional en el que desarrollan su actividad han permitido comprender mejor la dinámica reciente de los destinos turísticos de sol y playa, como es el caso de Fuerteventura. No obstante, para finalizar, deben señalarse alguna limitación en este trabajo. Y es que la propia condición de estudio de caso obliga a tomar con precaución las conclusiones alcanzadas. Pudiera ser que éstas se deriven más de las concretas condiciones del sector turístico de la isla de Fuerteventura que del sector turístico en general. Por lo que parece aconsejable que se lleven a cabo más estudios en esta dirección. 


\section{BIBLIOGRAFÍA}

AGARWAL, S. (2002): «Restructuring seaside tourism: The resort lifecyle», Annals of Tourism Research, vol. 29 (1), pp. 25-55.

ALMEIDA GARCÍA, F. (2012): «La política turística en España y Portugal», Cuadernos de Turismo, $\mathrm{n}^{\circ}$ 30, pp. 9-34.

ANDREWS, T.M. y W FRNESS, K. (2011): «Deprofessionalization of a female occupation: Challenges for the sociology of professions», Current Sociology, vol. 59 (1), pp. 42-58.

ARJONA GARRIDO, Á. y CHECA OLMOS, J.C. (2006a): «Economía étnica. Teorías, conceptos y nuevos avances», Revista Internacional de Sociología, vol. 64 (45), pp. 117-143.

ARJONA GARRIDO, Á. y CHECA OLMOS, J.C. (2006b): «Empresariado extracomunitario en Almería: estructuras de oportunidad, características de grupo y estrategias étnicas», Revista Española de Investigaciones Sociológicas, nº 115, pp. 297-317.

ÁVILA BERCIAL, R. y BARRADO TIMÓN, D.A. (2005): «Nuevas tendencias en el desarrollo de destinos turísticos: marcos conceptuales y operativos para su planificación y gestión», Cuadernos de Turismo, $\mathrm{n}^{\circ}$ 15, pp. 27-44.

BECERRA RODRÍGUEZ, F. (2008): «Las redes empresariales y la dinámica de la empresa: aproximación teórica», Revista Innovar, ${ }^{\circ} 18$ (32), pp. 27-46.

BENSON, M. y O'REILLY, K. (2009): «Migration and the search for a better way of life: a critical exploration of lifestyle migration», The Sociological Review, vol. 57 (4), pp. 608-625.

BRUNET ICART, I. y GALEANA FIGUEROA, E. (2013): «El nuevo paradigma empresarial: el esquema de redes», Revista EAN, n ${ }^{\circ}$ 50, pp. 26-39.

CASANUEVA ROCHA, C. ET AL. (2010): «Capital social e innovación en clústers industriales», Revista Europea de Dirección y Economía de la Empresa, vol. 19 (4), pp. 37-58.

COOPER, C. y HALL, C. M. (2008): Contemporary tourism: an international approach. Routledge.

DÍAZ RODRÍGUEZ, P. ET AL. (2012): «Selección patrimonial: del consumo cotidiano al consumo turístico (isla de Fuerteventura, Islas Canarias», Revista Andaluza de Antropología, $\mathrm{n}^{\circ}$ 2, pp. 74-95.

DIEZ, J.I. (2008): «Organizaciones, redes, innovación y competitividad territorial: análisis del caso Bahía Blanca», Redes. Revista hispana para el análisis de redes sociales, $\mathrm{n}^{\circ}$ 14.

DREDGE, D. (2006): «Policy networks and the local organisation of tourism», Tourism Management, vol. 27 (2), pp. 269-280.

ELORIE, F. (2009): «El papel de las redes sociales en la actividad económica: El caso de los restauradores de Lille», Redes: Revista Hispana para el análisis de redes sociales, $\mathrm{n}^{\mathrm{o}} 16$, pp. 202-227.

FRÄNDBERG, L. (2014): «Temporary transnational youth migration and its mobility links», Mobilities, vol. 9 (1), pp. 146-164.

GARCÍA MACÍAS, A. (2002): «Redes sociales y ‘clusters’ empresariales», Redes: Revista Hispana para el análisis de redes sociales, vol. 1, pp. 1-20. 
GIL, V. y ROMERO, F. (2008): Crossumer. Claves para entender al consumidor de nueva generación. Barcelona, Ediciones Gestión 2000.

GINER MONFORT, J. (2013): «Sorry, I'm not a Tourist: migración y turismo en La Marina Alta», Papers de Turisme, $\mathrm{n}^{\circ}$ 54, pp. 139-155.

GONZÁLEZ ENRÍQUEZ, C. (2008): «Los otros inmigrantes: los europeos comunitarios de países ricos», Boletín Elcano, n ${ }^{\circ} 106$.

GONZÁLEZ, M. y RUIZ, D. (2006): «La competitividad internacional de los destinos turísticos: del enfoque macroeconómico al enfoque estratégico», Cuadernos de Turismo, $\mathrm{n}^{\circ} 17, \mathrm{pp} .7-24$.

HERRERA ECHEVERRI, H. (2009): «Investigación sobre redes sociales y emprendimiento: revisión de la literatura y agenda futura», Innovar, vol. 19 (33), pp. 19-33.

HUETE, R.; MANTECÓN, A. y ESTÉVEZ, J. (2013): «Challenges in lifestyle migration research: Reflections and findings about the Spanish crisis», Mobilities, vol. 8 (3), pp. 331-348.

IOANNIDES, D. y DEBBAGE, K. (1997): «Post-Fordism and flexibility: the travel industry polyglot», Tourism Management, vol. 18 (4), pp. 229-241.

JONKER, E. ET AL. (2009): «The role and attributes of entrepreneurs at South Africa's largest arts festival», Special Issue, Innovation and entrepreneurship in the tourism industry, vol. 7, pp. 381-392.

LARDIÉS, R. (1999): «Migration and tourism entrepreneurship: North-european immigrants in Cataluña and Languedoc», International Journal of Population Geography, vol. 5 (6), pp. 477-491.

LATIESA, M. y PANIZA, J.L. (2006): «Turistas deportivos. Una perspectiva de análisis», Revista Internacional de Sociología, vol. 66 (44), pp. 133-149.

MARTÍN ARTILES, A. ET AL. (2011): «Movilidad ascendente de la inmigración en España: ¿asimilación o segmentación ocupacional?», Papers: Revista de Sociología, vol. 96 (4), pp. 1.335-1.362.

MARTÍN ROJO, I. y PELÁEZ VERDET, A. (2006): «Nuevos productos turísticos: El turismo activo. Diagnóstico empresarial en la provincia de Málaga», Estudios Turísticos, $\mathrm{n}^{\circ} 169$, pp. 145-163.

MEMBRADO TENA, J.C. (2015): «Migración residencial y urbanismo expansivo en el Mediterráneo español», Cuadernos de Turismo, n 35, pp. 259-286.

MERINERO RODRÍGUEZ, R. (2010): «Desarrollo local y análisis de redes sociales: El valor de las relaciones como factor del desarrollo socioeconómico», Redes: Revista Hispana para el análisis de redes sociales, vol. 18 (1), pp. 278-304.

MORALES, A.G. ET AL. (2012): «El desarrollo urbano turístico de Fuerteventura: la búsqueda del desarrollo sostenible versus al crecimiento constructivo masivo», Boletín de la Asociación de Geógrafos Españoles, n 59, pp. 7-24.

MUZIO, D. y KIRKPATRICK, I. (2011): «Introduction: Professions and organizations - a conceptual framework», Current Sociology, vol. 59 (4), pp. 389-405.

OLIVEIRA ARRUDA DE D. ET AL. (2014): «Coordinación y estructuras de gobernanza en un sistema productivo de turismo», Estudios y Perspectivas en Turismo, vol. 23 (2), pp. 343-361. 
O'REILLY, K. (2012): «Structuration, practice theory, ethnography and migration». International Migration Institute Working Papers Series.

O'REILLY, K. (2000): The British on the Costa del Sol: Transnational identities and local communities. Psychology Press.

OSO, L. (2012): «Inmigración, género y mercado de trabajo: una panorámica de la investigación sobre la inserción laboral de las mujeres inmigrantes en España», Cuadernos de Relaciones Laborales, vol. 30 (1), pp. 11-44.

PETERS, M. ET AL. (2009): «The importance of lifestyle entrepreneurship: A conceptual study of the tourism industry», Pasos, vol. 7 (2), pp. 393-405.

RATH, J. y KLOOSTERMAN, R. (2000): «Outsiders» business: A critical review of research on immigrant entrepreneurship», International Migration Review, vol. 34 (3), pp. 657-681.

RILEY, M. ET AL. (2002): Tourism employment. Analysis and planning. Channel View Publications. England.

SÁEZ CARRERAS, J. (2005): «La profesionalización de los educadores sociales: construcción de un modelo teórico para su estudio», Revista de Educación, n 336, pp. 129-139.

SANTANA TALAVERA, A. ET AL. (2011): «Renovación de destinos y percepción de la protección ambiental: el caso de Fuerteventura (Islas Canarias, España)», Investigaciones Turísticas, $\mathrm{n}^{\circ} 1$, pp. 1-20.

SANTANA TALAVERA, A. ET AL. (2015): «Avances sobre la gobernanza y el turismo en la Reserva de Biosfera de Fuerteventura (Islas Canarias)», en Governança e Turismo. Editorial ISMAI, pp. 3-37.

SCIULLI, D. (2007): «Professions before Professionalism», European Journal of Sociology / Archives Européennes de Sociologie, vol. 48 (1), pp. 121-147.

SIMÔES BRASILEIRO, M.D. (2006): El deporte y el turismo de litoral: entre los cambios sociales. Editorial Universidad de Granada. Granada.

SMERAL, E. (1998): «The impact of globalization on small and medium enterprises: new challenges for tourism policies in European countries», Tourism Management, vol. 19 (4), pp. 371-380.

SOLÉ, C. ET AL. (2000): «El impacto de la inmigración en la sociedad receptora», Revista Española de Investigaciones Sociológicas, no 90, pp. 131-157.

STONE, I. y STUBBS, C. (2007): «Enterprising expatriates: Lifestyle migration and entrepreneurship in rural Southern Europe», Entrepreneurship and Regional Development, vol. 19 (5), pp. 433-450.

SOLÉ, C. ET AL. (2007): El empresariado inmigrante en España. Fundación La Caixa.

VELASCO GONZÁLEZ, M. (2005): «¿Existe la política turística? La acción pública en materia de turismo en España (1951-2004)», Política y sociedad, vol. 42 (1), pp. 169-195.

VERA REBOLLO, J.F. y BAÑOS CASTIÑEIRA, C.J. (2010): «Renovación y reestructuración de los destinos turísticos consolidados del litoral: las prácticas recreativas en la evolución del espacio turístico», Boletín de la Asociación de Geógrafos Españoles, ${ }^{\circ}$ 53, pp. 329-353. 
WARDE, A. (2005): «Consumption and theories of practice», Journal of Consumer Culture, vol. 5 (2), pp. 131-153.

WILLIAMS, A.M. y SHAW, G. (2011): «Internationalization and innovation in tourism», Annals of Tourism Research, vol. 38 (1), pp. $27-51$. 